\title{
Manifiesto Abarán: el farmacéutico comunitario en la atención domiciliaria y sociosanitaria
}

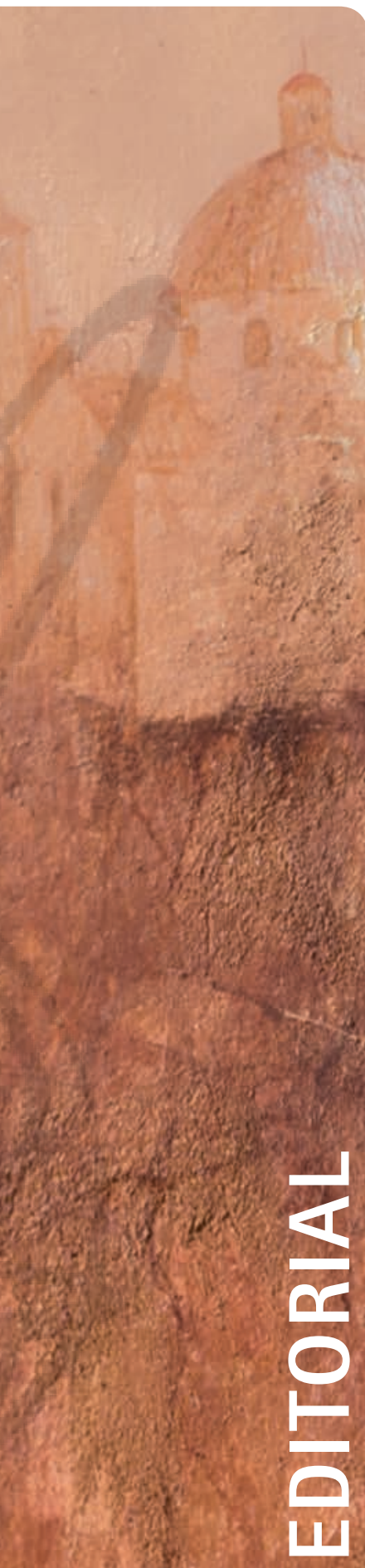

En los últimos años la esperanza de vida en España ha aumentado considerablemente, de modo que actualmente el 18 por ciento de la población es mayor de 65 años (los mayores de 80 años son el 6 por ciento) y con previsiones de que en solo quince años una de cada cuatro personas superará esa edad. Algunas estimaciones apuntan, incluso, a que España será el tercer país más envejecido del mundo en 2050.

A esto hay que añadir que a medida que la vida de las personas avanza suelen ir apareciendo enfermedades crónicas que, poco a poco, van reduciendo su calidad de vida, de tal manera que al llegar a la vejez es frecuente que se den varias patologías y que ello produzca un estado de fragilidad y dependencia. Además, y aunque según los datos disponibles el 72 por ciento de los mayores de 65 años son pacientes con patologías crónicas, no se debe olvidar que la cronicidad no está exclusivamente ligada a la edad, sino que afecta también a población infantil y juvenil.

Asimismo, las personas mayores expresan una clarísima voluntad de autonomía, como manifiesta el hecho de que un 87 por ciento desee vivir en sus casas el mayor tiempo posible. Sería deseable que todos respetáramos el derecho a la autonomía y elección de las personas mayores sobre dónde y cómo quieren vivir.

Es sin duda en esta etapa cuando, además de que el paciente reciba cuidados de la máxima calidad, es importante mantener un entorno que le resulte familiar y de confianza. Es decir, lo más cercano posible y con profesionales sanitarios que tengan capacidad para prestar especial atención o derivar ante cualquier descompensación que se pueda dar en la salud del paciente, y que den una respuesta eficaz que, por su carácter pluripatológico, debe estar muy bien coordinada.

Por lo tanto, el aumento progresivo de la cronicidad, ligado al incremento de la esperanza de vida, el envejecimiento poblacional, la pluripatología, la polimedicación y la dependencia asociadas, exige cambios de estrategia y de reorganización encaminados a la creación de un espacio de atención sociosanitaria. Es en este espacio donde deben converger de forma, no solo coordinada, sino también simultánea y continuada, los cuidados de salud y de atención social que no solo contribuyan a curar sino especialmente a cuidar a todas aquellas personas con problemas de salud crónicos, dependencia, dificultad para desplazarse de su domicilio y/o de riesgo de exclusión social. Respecto al uso de los medicamentos, también hay que tener en cuenta que un considerable porcentaje de la población (más de un 40 por ciento) reconoce tener carencias informativas sobre sus tratamientos, lo que si ya de por sí merece una actuación proactiva por parte del farmacéutico comunitario para paliar este déficit, todavía es más importante en el ámbito de los pacientes especialmente vulnerables.

En este contexto, tampoco se debe olvidar que en la actualidad hay en España 4,5 millones de personas que viven solas. Esta cifra previsiblemente aumentará en el futuro, y se puede agravar por la ausencia del soporte familiar derivado de los cambios en el modelo familiar, lo que exigirá un refuerzo de la estructura de colaboración sociosanitaria.

Adaptar los servicios sanitarios para proveer al paciente con patologías crónicas y/o dependiente de los cuidados adecuados a sus necesidades se ha convertido en el mayor reto al que se enfrenta la sanidad actual. Todos los modelos de atención al paciente con patologías crónicas coinciden en señalar que: 
- Los cuidados sanitarios han de proveerse en el entorno del paciente $\mathrm{y}$, en concreto, tan cerca de su domicilio como sea posible. Este aspecto por sí solo obliga a reorientar por completo las prioridades de los recursos y servicios sanitarios, desplazando el eje de la atención hospitalaria a la primaria y potenciando su extensión hacia la atención domiciliaria.

- Una atención adecuada debe incluir el empoderamiento del paciente para que se involucre en el control de la enfermedad, de forma que las decisiones que adopte (tomar o no la medicación, mantener o no hábitos adecuados para su salud, etc.) sean correctas. Para conseguir esa implicación del paciente se necesitan profesionales sanitarios en los que el paciente confíe.

- Debe identificarse el momento en que el paciente empieza a tener un mal control de su enfermedad para poder así actuar antes de que se produzcan consecuencias clínicas negativas. Por tanto, es necesario generar una red sanitaria que supervise de cerca la evolución del paciente y de la que el farmacéutico comunitario no sea ajeno.

- Los cuidados han de ser multidisciplinares, y abarcar tanto cuidados sanitarios como sociosanitarios que cubran los aspectos fisiopatológicos y los determinantes sociales de la enfermedad. Ello plantea el requisito, especialmente complejo, de que los distintos profesionales sean capaces de trabajar juntos de forma coordinada.

\section{¿Qué papel juega el farmacéutico comunitario?}

1. El farmacéutico comunitario debe ser responsable de las necesidades farmacoterapéuticas de los pacientes que no estén ingresados en un hospital independientemente de dónde vivan. El farmacéutico comunitario, tanto por sus conocimientos farmacológicos como por su accesibilidad y cercanía es, hoy por hoy, el profesional sanitario que posee una visión más global del tratamiento farmacológico (especialmente complejo en pacientes pluripatológicos) y forma de vida del paciente. Históricamente la población siempre ha manifestado una gran confianza en el farmacéutico comunitario y desde la introducción de la receta electrónica es el sanitario al que el paciente visita con más frecuencia; hecho que le permite conocer los medicamentos de prescripción y de automedicación que utiliza cada paciente.

2. El vínculo establecido entre la población y el farmacéutico comunitario, la confianza mutua y el conocimiento que el farmacéutico comunitario tiene del entorno familiar y social del paciente, le convierte en un profesional necesario para conseguir el empoderamiento y la implicación del paciente.

3. El farmacéutico comunitario debe formar parte de los equipos de atención primaria, junto a médicos, enfermeros y trabajadores sociales, como proveedor de atención sanitaria a los pacientes más vulnerables sobre todos aquellos con mayor falta de autonomía o con patologías crónicas.

4. Los más de 45.000 farmacéuticos comunitarios que ejercen actualmente en España, distribuidos uniformemente en todo el territorio en 22.000 farmacias, deben formar parte de la red de supervisión que detecte riesgos de descompensación en la salud de los pacientes con patologías crónicas para, de este modo, actuar con celeridad y evitar complicaciones de gravedad.

\section{Compromisos SEFAC}

Por todo lo anterior la Sociedad Española de Farmacia Familiar y Comunitaria (SEFAC) se compromete con los pacientes, la profesión farmacéutica y la sociedad a promover y facilitar la integración del farmacéutico comunitario en la atención del paciente ambulatorio o no hospitalizado, y en especial de aquellas personas que tengan especiales dificultades para acceder a un centro sanitario. Para ello se asumen los siguientes compromisos:

1. Potenciar la figura del farmacéutico comunitario como profesional sanitario de referencia en la asistencia farmacoterapéutica del paciente.

2. Liderar la formación y capacitación de los farmacéuticos comunitarios para adecuar sus competencias y habilidades profesionales a las necesidades de los pacientes.

3. Promover el trabajo conjunto con Administraciones públicas y privadas, asociaciones de pacientes, sociedades científicas y colegios profesionales con el fin de lograr la mejor atención integral del paciente.

4. Coordinar las actuaciones del farmacéutico comunitario con el resto de profesionales que forman parte del equipo de atención al paciente.

5. Desarrollar la prestación de los servicios profesionales farmacéuticos necesarios para aquellos pacientes no hospitalizados y que por sus circunstancias de salud, discapacidad o condicionantes sociofamiliares no puedan recibirlos fuera de su lugar de residencia.

6. Impulsar la educación para la salud y la prevención en materia de patologías crónicas desde la farmacia comunitaria.

7. Apoyar el desarrollo e implantación de herramientas que faciliten la atención farmacéutica integrada en la actividad diaria de la farmacia.

8. Colaborar con las escuelas, foros y/o asociaciones de pacientes $\mathrm{u}$ otras fórmulas de apoyo destinadas a los cuidadores de los pacientes crónicos.

9. Coordinar investigaciones que midan el impacto en salud y el coste-efectividad de los modelos de atención al paciente con patología crónica que incluyan al farmacéutico comunitario como parte de un equipo multidisciplinar.

10. Generar indicadores de calidad de los procesos de atención al paciente frágil, crónico y polimedicado e integrar las nuevas tecnologías en su cuidado y seguimiento.

Abarán (Murcia), 6 de marzo de 2015 Sociedad Española de Farmacia Familiar y Comunitaria 